\title{
AOTrauma D-A-CH-Reisestipendium 2018
}

Hauke Rüther

Im Januar 2019 erhielt ich aufgrund der großzügigen Unterstützung durch die AOTrauma Deutschland die Chance, für 14 Tage im Inselspital Bern zu hospitieren. Für mich bot sich so die einmalige Chance, bei einem der führenden Kindertraumatologen/-orthopäden, Herrn PD Dr. Ziebarth, lernen zu dürfen. Vor allem hat sich das Berner Spital über Jahre in der Weiterentwicklung der Versorgung der kindlichen Frakturen in der AO sowie im Bereich der Hüftchirurgie hervorgetan. Vorab wurde schnell klar, dass die Abteilung eine große Erfahrung mit Fellowships hat. Dank der perfekten Organisation durch das Team und Herrn Steffen, dem Chefarztsekretär, war die Vorabplanung und Ankunft in Bern völlig unkompliziert.

Die Kindertraumatologie und -orthopädie ist in die Kinderchirurgie des Inselspitals eingegliedert. Die Kinderklinik ist in Gänze in einem separaten Gebäude mit eigenem OP-Trakt und Kinderintensivstation untergebracht. Strukturell merkt man gleich die Vorteile dieser Untergruppierung durch die kinderfreundliche Gestaltung der Räumlichkeiten und die somit auch vorhandene Spezialisierung auf die besondere Klientel. Die Kinderchirurgie wird durch Herrn Prof. Berger geleitet, wobei die Kindertraumatologie und -orthopädie in den Händen von Herrn PD Dr. Ziebarth liegt. In seinem Team arbeiten 2 Oberärzte (Herr PD Dr. Liebs und Frau Dr. Kaiser) sowie weiterhin als Konsiliararzt Herr Dr. Slongo, der bis 2013 die Kinderorthopädie leitete und sich jahrzehntelang in der Kindertraumatologie in der AO hervortat. Neben diesen Kollegen sind im Wechsel Fach- und allgemeine Assistenten tätig.

Ab dem 1. Tag wurde ich vom gesamten Team überaus herzlich empfangen. Von der gesamten Abteilung wurde mir direkt das in der Schweiz üblich „Du“ angeboten und es fand ein reger Austausch statt. Die große Expertise und konsequente Umsetzung der AO-Prinzipien wurde rasch ersichtlich.

Nach Ankunft gegen 7:00 h wurde ich zum Morgenrapport begleitet. Hier kam mir im Rahmen der Falldemonstration vom Wochenende rasch die Frage: „Alles Wintersport oder was?“. Die hohe Frequenz mit typischen Frakturen wurde deutlich. Vom letzten Wochenende, welches als überaus ruhig beschrieben wurde, waren allein 3 Unterschenkelfrakturen nach Skiunfall vorhanden.
Hier imponierte mir vom ersten Moment der dezidierte Umgang mit den einzelnen Pathologien unter genauer Kenntnis der Risiken und des jeweiligen Potenzials zur Korrektur. Denn nicht nur das „Messer wetzen“ steht hier im Vordergrund, sondern auch die Kunst, Frakturen, die dies zulassen, adäquat konservativ zu behandeln. Wichtig hierbei war Herrn PD Dr. Ziebarth zu jeder Zeit die vernünftige Kommunikation mit den Eltern und, wo es möglich war, das Einbinden dieser in die Entscheidungsfindung. Denn bei einer konservativen Therapie, bei der für einige Zeit zwar eine Deformität ersichtlich ist, welche sich jedoch mit dem Wachstum korrigiert, ist es extrem wichtig, dass die Familie diesen Weg mitträgt und nicht völlig verängstigt die Extremität des Kindes betrachtet. Im Rahmen der konservativen Therapie konnte ich so mehrmals die vielerorts verlernte Gipskeilung begutachten und auch selbst Hand anlegen.

Dies soll jedoch nicht heißen, dass in Bern nur Gips und Softcast stattfindet. Denn der OP ist ausgebucht. Während meiner 2 Wochen konnte ich die ganze Expertise der operativen Versorgung erleben. Wir begannen mit den nahezu alltäglichen ESIN-Versorgungen von Extremitäten und Kreuzbandplastiken, die aufgrund der Nähe zum Skigebiet jede Woche vorkommen. Dann kam es bereits in der 1 . Woche zu 2 Raritäten. Zunächst stellte sich ein 11-jähriger Junge vor, der bei einem Bodycheck beim Eishockeyspielen an der Bande eine grob dislozierte Claviculafraktur erlitten hatte. Hier drohte eine Durchspießung trotz Rucksackverband, sodass wir eine ESIN-Osteosynthese von medial durchführten.

Nach so vielen Skiunfällen kam schon die Frage auf, ob es denn nichts anderes hier geben würde. Doch da kreuzte ein Altbekannter unsere Wege. Der Reitunfall, jedoch mit der 2. Rarität: Ein 12-jähriges Mädchen hatte eine Schenkelhalsfraktur erlitten. Hier wurde mir dann das erste Mal bewusst, dass in Bern eine große Expertise und jahrelange Erfahrung in der Hüftchirurgie vorherrscht, denn die Fraktur wurde in beindruckender Leichtigkeit offen reponiert und mittels Pediatric Hip Plate versorgt. Das 2. Mal durfte ich dann die Erfahrung in der Hüftchirurgie bei einem 14 Monate alten Mädchen mit Hüftdysplasie erleben. Es erfolgte über einen Smith-Peterson-Zugang beidseits in minutiöser Präparation, unter genauer Kenntnis der Fallstricke dieser OP und der anatomischen Variationen, die offene Reposition und Kapselraffung. 


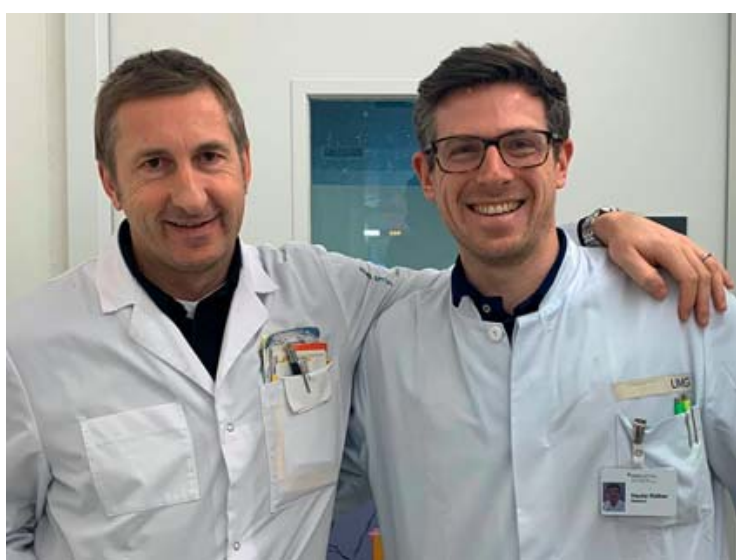

Abb. 1 Kai Ziebarth und Hauke Rüther. Quelle: Hauke Rüther

Während des weiteren Aufenthaltes erfolgten auch noch die typischen kindlichen Osteosynthesen.

Im Rahmen der Sprechstunden konnte ich im Weiteren sehr viel über den Umgang mit Korrekturgrenzen und dem schmalen Grat zwischen konservativem und operativem Vorgehen lernen. Hier wurden auch mehrere Kinder vorstellig, die mit sekundären Deformitäten nach Trauma zugewiesen wurden; gemeinsam mit den Familien wurde ein „Schlachtplan“ entwickelt, der für die gesamte Familie nachvollziehbar und mitzutragen war. Leider musste ich hier aber auch die Grenzen des Machbaren und die manchmal schicksalhaften Verläufe miterleben, die im Kinderbereich noch tragischer sind.

Ebenfalls teilnehmen durfte ich an der Nachsorge eines Jungen mit Osteosarkom, bei dem zuvor die En-bloc-Resektion im Bereich des distalen Oberschenkels mit Kniegelenk erfolgt war und in interdisziplinärer OP eine Umkehrplastik durchgeführt wurde. Hier zeigte sich ein sehr schönes Ergebnis und ein den Umständen entsprechend zufriedener Junge.

Im Rahmen des kollegialen Austausches und der Demonstration der beeindruckenden Fallsammlung kam es durch Zufall dazu, dass an beiden Kliniken nach transkondylärer distaler Humerusfraktur beim Adoleszenten und regelrechter Metallentfernung eine Refraktur aufgetreten war. Dies brachte uns dazu, eine Literaturrecherche

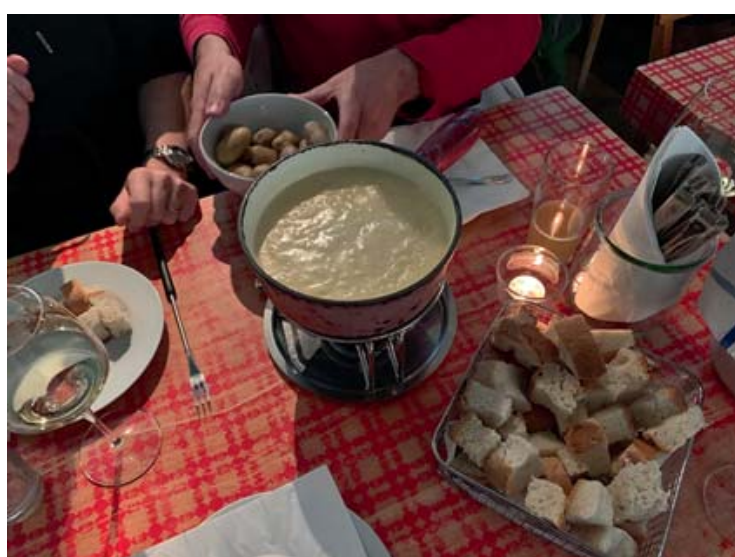

- Abb. 2 Typisches Schweizer Käsefondue. Quelle: Hauke Rüther

durchzuführen und aufgrund der Rarität der Verletzung nun einen bizentrischen Case Report zu schreiben.

Während der Hospitation konnte ich des Weiteren die wunderschöne Berner Stadt besichtigen und aufgrund der Tipps der Mitarbeiter viele schöne Gegenden und Sehenswürdigkeiten besuchen. Abgerundet wurde der Besuch durch einen geselligen Abend mit typischem Käsefondue und weiteren Speisen und Getränken.

Abschließend bleibt zu sagen, dass Bern in jeder Hinsicht eine Reise wert ist. Der Aufenthalt war für meine fachliche und persönliche Entwicklung mehr als gewinnbringend. Ich konnte hier viele Dinge sehen und lernen und inzwischen auch einiges im klinischen Alltag anwenden. Für die Gelegenheit möchte ich mich insbesondere bei Herrn PD Dr. Ziebarth und der AOTrauma Deutschland bedanken.

Korrespondenzadresse

\section{Hauke Rüther}

Universitätsmedizin Göttingen

hauke.ruether@med.uni-goettingen.de

\section{Bibliografie}

DOI https://doi.org/10.1055/a-0948-6103

OP-JOURNAL 2019; 35: 209-210 @ Georg Thieme Verlag KG Stuttgart · New York ISSN 0178-1715 